\title{
Incipient Non-Arteritic Anterior Ischemic Optic Neuropathy in a Patient with Metastatic Small-Cell Lung Cancer
}

\author{
Arshia Eshtiaghi ${ }^{a} \quad$ Jonathan A. Micieli $b, c, d$ \\ ${ }^{a}$ Faculty of Medicine, University of Toronto, Toronto, ON, Canada; ${ }^{b}$ Department of \\ Ophthalmology and Vision Sciences, University of Toronto, Toronto, ON, Canada; 'Division \\ of Neurology, Department of Medicine, University of Toronto, Toronto, ON, Canada; \\ ${ }^{\mathrm{d} K e n s i n g t o n}$ Vision and Research Centre, Toronto, ON, Canada
}

\section{Keywords}

Incipient non-arteritic anterior ischemic optic neuropathy - Optic disc edema - Ischemia .

Metastatic cancer · Hypercoagulability

\begin{abstract}
A 70-year-old woman with metastatic small-cell lung cancer was referred for isolated left optic disc edema that was incidentally discovered. She had normal visual function, and dilated fundus examination revealed a small, cupless optic nerve in the right eye and moderate optic disc edema in the left eye. Magnetic resonance imaging (MRI) of the orbits with contrast was normal, and MRI brain and magnetic resonance venography were normal without signs of raised intracranial pressure. Lumbar puncture showed a normal opening pressure and normal cerebrospinal fluid contents. A diagnosis of incipient non-arteritic anterior ischemic optic neuropathy (NAION) was made, and the optic disc edema resolved after 4 months. Incipient NAION is an uncommon cause of unilateral optic disc edema with preserved visual function and is a diagnosis of exclusion. In diagnosing incipient NAION, other causes of optic disc edema must first be ruled out. These alternative causes include papilledema, optic nerve sheath meningioma or other orbital masses, and vitreopapillary traction. Incipient NAION is thought to be caused by subclinical ischemia. This case indicates that incipient NAION may also occur in patients with metastatic cancer and is possibly related to their hypercoagulable state. Although there is no treatment once vision loss develops, the optimization of risk factors may prevent the progression of incipient NAION to classic NAION.
\end{abstract}

(C) 2021 The Author(s).

Published by S. Karger AG, Basel 


\section{Introduction}

Patients with metastatic cancer may present with neuro-ophthalmic signs and symptoms, which may be directly or indirectly related to the cancer itself, the treatment of the cancer, or may be unrelated to the underlying cancer diagnosis [1]. Non-arteritic anterior ischemic optic neuropathy (NAION) has rarely been described in patients with metastatic disease. It is important to include NAION in the differential diagnosis as optic neuropathies may be incorrectly attributed to cancer treatment, resulting in the cessation of important care [2]. NAION classically involves a history of sudden, painless vision loss accompanied by optic disc edema in patients with a "disc-at-risk." Uncommonly, patients will present with a clinical pattern that is consistent with NAION, but without any subjective or objective loss of vision. This is termed "incipient" NAION and is often a diagnosis of exclusion that first involves exclusion of other causes such as a retrobulbar mass such as an optic nerve sheath meningioma or unilateral papilledema [3]. A previous case series of 54 patients found that $25 \%$ of people with incipient NAION progressed to develop a visual field defect after a median of 5.8 weeks, and another $20 \%$ developed a visual field defect after the resolution of the first episode of incipient NAION [4]. The early recognition of incipient NAION may allow clinicians to mitigate risk factors and prevent progression to classic NAION. Here, we present a case of a 70-year-old woman with metastatic small-cell lung cancer who is incidentally found to have left optic disc edema that was later diagnosed as incipient NAION.

\section{Case Report/Case Presentation}

A 70-year-old woman was referred by her optometrist after she was noted to have left optic disc edema on a routine examination. She had a past medical history of metastatic smallcell lung cancer that was diagnosed 1 year after she was found to have a right lower lobe pulmonary mass with associated post-obstructive atelectasis, a liver mass, and bone metastasis. She completed 6 cycles of palliative carboplatin and etoposide treatment 6 months prior to presentation. She also underwent chest radiation and was treated with maintenance atezolizumab. Her past medical history also included idiopathic thrombocytopenia, asthma, mild Crohn's disease, gastroesophageal reflux disease, restless leg syndrome, and dyslipidemia. Her medications included gabapentin, pantoprazole, ferrous fumarate, rosuvastatin, and pramipexole.

At her initial neuro-ophthalmology consultation, she denied blurred vision, pain, or headaches. Visual acuity was 20/25 in both eyes, pupils were equally reactive, and there was no relative afferent pupillary defect; Humphrey 24-2 SITA-Fast visual fields were normal in both eyes. Dilated fundus examination revealed a normal appearing optic nerve in the right eye (OD) and optic disc edema in the left eye (OS; shown in Fig. 1). Optical coherence tomography (OCT) of the retinal nerve fiber layer showed an average thickness of $107 \mu \mathrm{m}$ OD and $356 \mu \mathrm{m}$ OS. High definition OCT over the optic nerve showed no signs of vitreopapillary traction (shown in Fig. 2). The erythrocyte sedimentation rate was mildly elevated at $43 \mathrm{~mm} / \mathrm{h}$, C-reactive protein was normal $(4.5 \mathrm{mg} / \mathrm{L}$, normal $<5.0 \mathrm{mg} / \mathrm{L})$, and the patient had no symptoms related to giant cell arteritis. The mildly elevated erythrocyte sedimentation rate was attributed to her metastatic cancer.

Since the patient had left optic disc edema with preserved visual function, the differential diagnosis considered was a retrobulbar mass such as a metastasis or optic nerve sheath meningioma, unilateral papilledema, or incipient NAION. Vitreopapillary traction had been ruled out with OCT of the optic nerve.

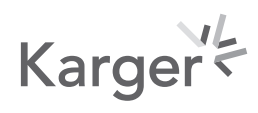




\section{Case Reports in Ophthalmology}

\begin{tabular}{l|l}
\hline Case Rep Ophthalmol 2021;12:513-518 \\
\hline DOI: 10.1159/000516573 & $\begin{array}{l}\text { @ 2021 The Author(s). Published by S. Karger AG, Basel } \\
\text { www.karger.com/cop }\end{array}$ \\
\hline
\end{tabular}

Eshtiaghi and Micieli: Incipient NAION in Patient with Metastatic Small-Cell Lung Cancer

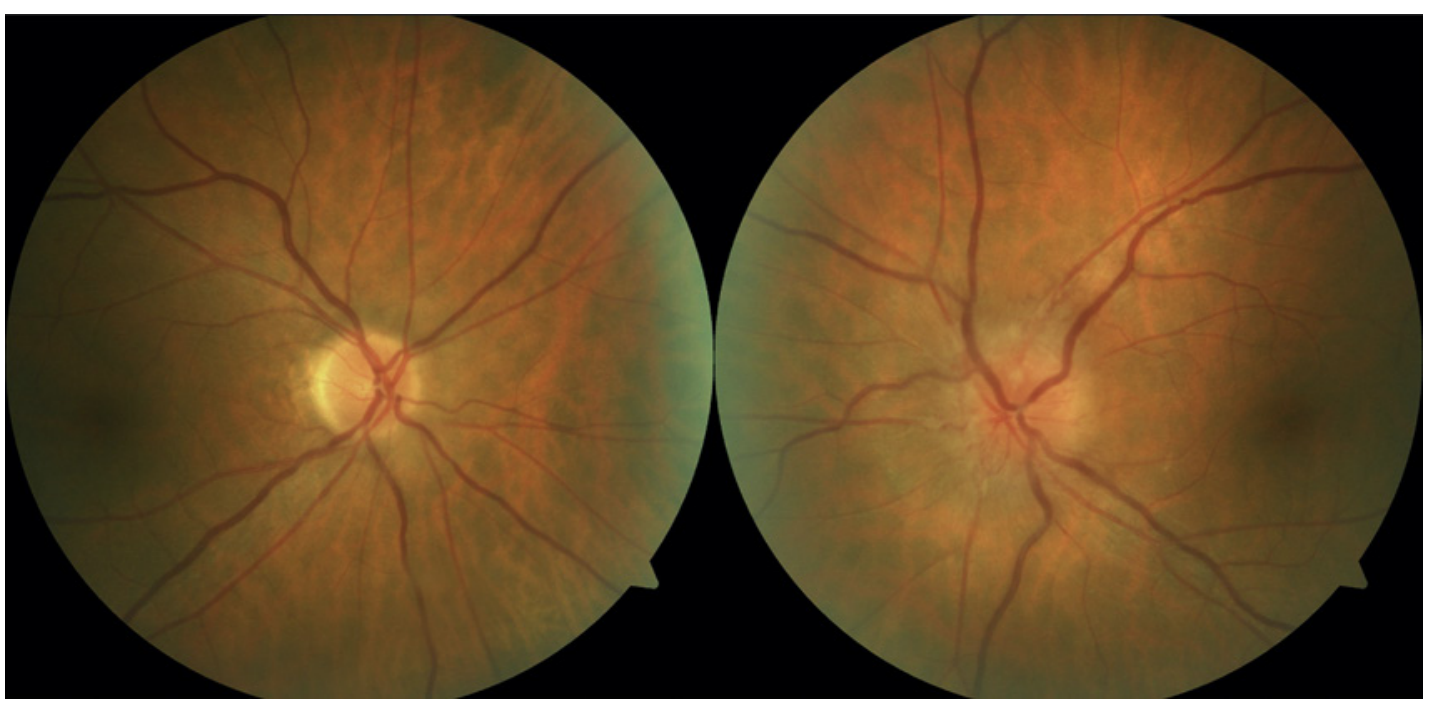

Fig. 1. Optic disc photos demonstrating a normal right optic nerve with a small cup-to-disc ratio and left optic disc edema.
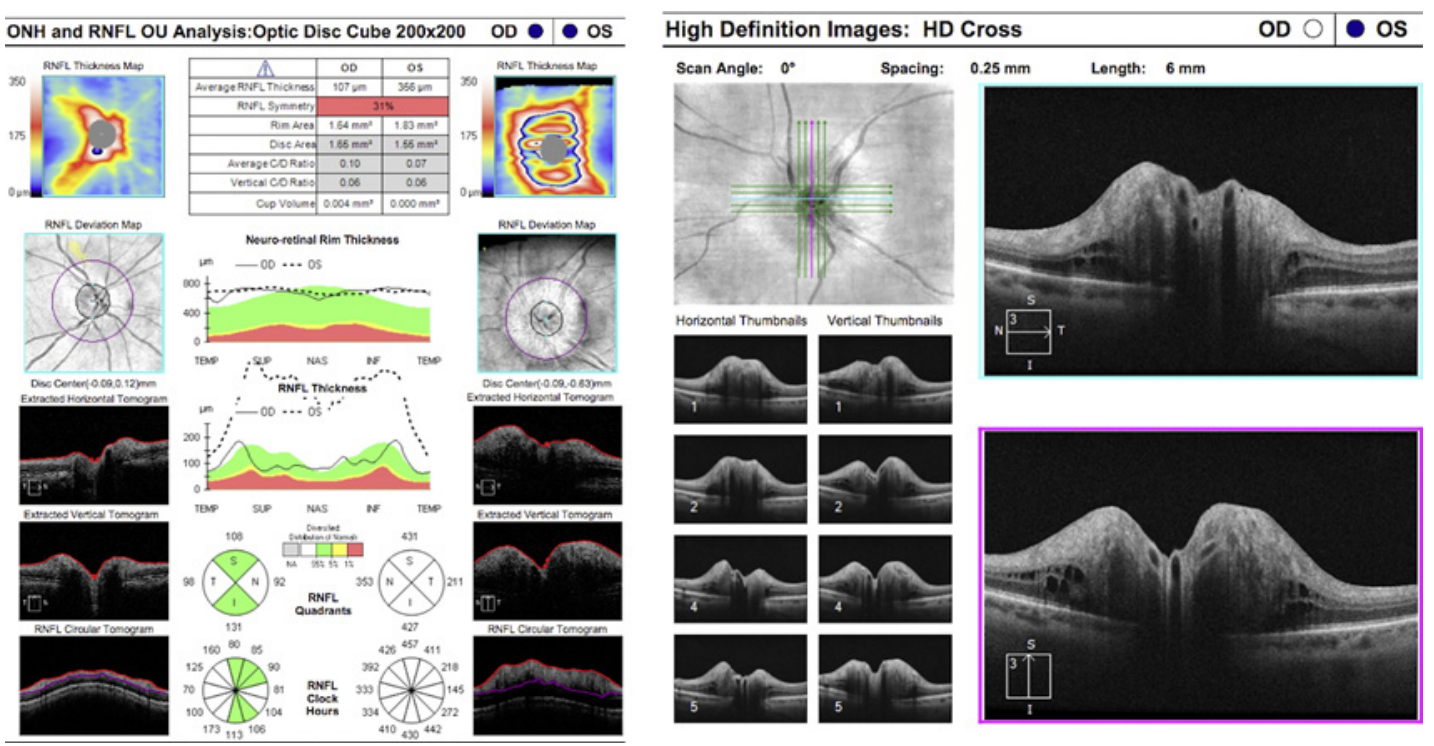

Fig. 2. OCT of the retinal nerve fiber layer showing an elevated RNFL thickness in the left eye (left panel). OCT of the optic nerve (HD cross) showing left optic disc edema without optic disc drusen or vitreopapillary traction (right panel). OCT, optical coherence tomography.

To narrow down the differential diagnosis, further investigations were performed. She underwent magnetic resonance imaging of the brain and orbits with contrast and magnetic resonance venography of the head, and this showed no orbital masses, no signs of leptomeningeal disease, and no signs of dural venous sinus thrombosis. There were no signs of raised intracranial pressure such as an empty sella or distal transverse sinus stenosis. She underwent a lumbar puncture that showed an opening pressure of $14 \mathrm{~cm}$ of water (normal less than 25 $\mathrm{cm}$ of water), and there was 1 nucleated cell and normal protein and glucose. Her hemoglobin was normal at $135 \mathrm{~g} / \mathrm{L}$.

Since a retrobulbar compressive mass raised intracranial pressure and vitreopapillary traction had been ruled out, a diagnosis of incipient NAION was made. As there is no estab- 
lished treatment for NAION, efforts were focused on modifying her risk factors. She saw her primary care physician to optimize her vascular risk factors and was started on aspirin $81 \mathrm{mg}$ daily. She had previously been tested for obstructive sleep apnea, which was normal.

The patient was followed up, and her left optic disc edema resolved at the 4-month follow-up visit. At that visit, her visual acuity continued to be $20 / 25$ in each eye, and Humphrey 24-2 SITA-Fast visual fields were normal. The spontaneous resolution of the optic disc edema confirmed the diagnosis of incipient NAION. She attended a follow-up appointment at 8 months and continued to maintain normal visual function without recurrence of the optic disc edema.

\section{Discussion/Conclusion}

While the exact mechanism of NAION remains controversial, it is generally presumed to result from acute ischemia to the optic nerve head, which then induces axonal swelling. In susceptible eyes with anatomically crowded optic discs, axons are compressed within the scleral canal, leading to compartment syndrome and retinal ganglion cell death [5]. The unique, asymptomatic presentation of incipient NAION is instead believed to be caused by subclinical ischemia [4]. Subclinical ischemia can produce optic disc edema without necessarily causing vision loss. Minor reductions in blood flow to the optic disc can cause axoplasmic flow stasis and edema without causing axonal cell death or interfering with visual impulse transmission. Given that up to $45 \%$ of eyes with incipient NAION eventually develop classic NAION, early intervention to prevent the progression of subclinical ischemia to severe ischemia may prevent permanent vision loss [4]. The risk factors for the development of NAION are predominantly modifiable and include obstructive sleep apnea, hypertension, hypercholesterolemia, and diabetes [6]. Therefore, it is recommended that any diagnosis of incipient NAION be followed up with prompt monitoring and treatment of these risk factors, if present. Once vision loss occurs, management still focuses on addressing these underlying risk factors, but there are no evidence-based treatments to prevent further deterioration. In terms of prognosis, Hayreh and Zimmerman reported the largest series of patients with this condition and found that $25 \%$ of patients with incipient NAION progressed to classical NAION after a median of 5.8 weeks, and $20 \%$ of patients developed classical NAION after resolution of the optic disc edema. The Ischemic Optic Neuropathy Decompression Trial found that in patients with classical NAION, $15 \%$ of patients will experience another episode of NAION in their fellow eye over a 5-year period $[4,7]$.

It is important to have a comprehensive differential diagnosis for optic disc edema in cancer patients since this may be misattributed to important cancer treatments. NAION in patients with cancer has been rarely reported. Yuan et al. reported a 48-year-old woman who experienced sudden vision loss after receiving her first cycle of intravenous chemotherapy with docetaxel, cisplatin, and fluorouracil for nasopharyngeal squamous cell carcinoma [8]. She underwent extensive investigations to exclude metastases, which were negative, and the vision did not improve. An additional case report documented NAION in a patient with colon cancer and optic disc drusen, which is also known to predispose to NAION [9]. Clinicians may sometimes misclassify NAION as "optic neuritis," especially in cancer patients on checkpoint inhibitors, which are known to increase the risk of inflammatory events [2]. Metastatic disease is also known to produce a hypercoagulable state throughout the body and can increase the risk of NAION. The secretion of procoagulant substances and inflammatory cytokines by cancer cells contributes to the formation of thrombi [10], which can occlude small blood vessels supplying blood to the optic nerves. Furthermore, cancer patients often develop anemia from inflammatory suppression of RBC production [11], which can further contribute to the ischemic state observed in NAION.

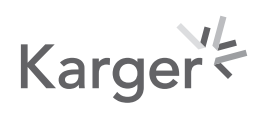


There is a specific differential diagnosis for optic disc edema with preserved visual function, and these cases should be evaluated in a systematic manner. For unilateral optic disc edema, this includes incipient NAION, unilateral papilledema from various causes (e.g., idiopathic intracranial hypertension, and dural venous sinus thrombosis), optic nerve sheath meningioma or similar orbital lesions, optic perineuritis, vitreopapillary traction, and peripapillary choroidal neovascular membrane [3]. For bilateral optic disc edema, the most common cause is papilledema, but other causes may include incipient NAION, optic perineuritis, intermediate uveitis, and a hypertensive emergency [12]. To rule out these alternative causes of optic disc edema, further examination with a slit lamp, dilated fundoscopy, optical coherence tomography, intravenous fluorescence angiography, magnetic resonance imaging, and magnetic resonance venography is often recommended. The diagnosis of incipient NAION is a diagnosis of exclusion that can be made once these other possibilities have been ruled out.

In summary, we present a case of a 70-year-old woman with metastatic small-cell lung cancer who was incidentally found to have left optic disc edema that was later diagnosed as incipient NAION. Before a diagnosis of incipient NAION can be made, it is essential to rule out other ocular, neoplastic, and systemic causes of optic disc edema. Once a diagnosis is made, careful monitoring of vasculopathic risk factors may decrease ischemia and prevent progression of vision loss. Further cases of NAION in patients with cancer are required to better characterize the disease in this setting.

\section{Acknowledgement}

This case report does not include any nonauthor contributors to acknowledge.

\section{Statement of Ethics}

Written informed consent was obtained from the patient for publication of this case report and accompanying images. The study protocol was reviewed, and the need for approval was waived by the University of Toronto Research Ethics Board. The article is exempt from ethical committee approval as it is a case report of a patient already previously seen. The case report adhered to the ethical standards of the Declaration of Helsinki.

\section{Conflict of Interest Statement}

The authors have no conflicts of interest to declare.

\section{Funding Sources}

This case report did not receive any funding.

\section{Author Contributions}

Jonathan A. Micieli and Arshia Eshtiaghi: conception and design of the work; acquisition, analysis, and interpretation of data for the work; and drafting and revising the work. 


\section{References}

1 Dermarkarian CR, Kini AT, Al Othman BA, Lee AG. Neuro-ophthalmic manifestations of intracranial malignancies. J Neuroophthalmol. 2020;40(3):e31-48.

2 Micieli JA, Margolin E. Re: Francis et al.: immune checkpoint inhibitor associated optic neuritis. Ophthalmology. 2020;127:1585-9.

3 Hawy E, Sharma RA, Peragallo JH, Dattilo M, Newman NJ, Biousse V. Unilateral isolated paucisymptomatic optic disc edema. J Neuro-Ophthalmology. 2020.

4 Hayreh SS, Zimmerman MB. Incipient Nonarteritic Anterior Ischemic Optic Neuropathy. Ophthalmology. 2007;114(9):P1763-72.

5 Levin LA, Louhab A. Apoptosis of retinal ganglion cells in anterior ischemic optic neuropathy. Arch Ophthalmol. 1996;114(4):488-91.

6 Cestari DM, Gaier ED, Bouzika P, Blachley TS, De Lott LB, Rizzo JF, et al. Demographic, systemic, and ocular factors associated with nonarteritic anterior ischemic optic neuropathy. Ophthalmology. 2016;123(12): P2446-55.

7 Newman NJ, Scherer R, Langenberg P, Kelman S, Feldon S, Kaufman D, et al. The fellow eye in NAION: report from the ischemic optic neuropathy decompression trial follow-up study. Am J Ophthalmol. 2002;134(3): P317-28.

8 Yuan X, Feng Y, Li D, Li M. Unilateral visual impairment in a patient undergoing chemotherapy: a case report and clinical findings. BMC Ophthalmol. 2019.

9 Elnahry AG. Anterior ischemic optic neuropathy in a patient with optic disc Drusen while on FOLFOX Chemotherapy for colon cancer: the value of Occam's Razor and Hickam's dictum. Rom J Ophthalmol. 2019;63(2): 174-7.

10 Caine GJ, Stonelake PS, Lip GYH, Kehoe ST. The hypercoagulable state of malignancy: pathogenesis and current debate. Neoplasia. 2002;4(6):465-73.

11 Adamson JW. The anemia of inflammation/malignancy: mechanisms and management. Hematology Am Soc Hematol Educ Program. 2008;2008(1):159-65.

$12 \mathrm{Yu} \mathrm{CW}$, Micieli JA. Bilateral optic disc edema with preserved visual function not related to papilledema. J Neurol Sci. 2020;418:117160. 